\title{
Nuevos documentos para el estudio de un convento de predicadores en la Oaxaca colonial: San Pedro Etla
}

\author{
Gloria Espinosa Spínola \\ Departamento de Historia, Geografía e Historia del Arte \\ Universidad de Almería
}

\begin{abstract}
El presente artículo estudia el convento de Predicadores de San Pedro en la ciudad de Etla, estado de Oaxaca, como ejemplo de los conventos rurales o vicarías que los dominicos establecieron en Nueva España. El trabajo, enfocado desde un punto de vista artístico, analiza las distintas etapas de construcción, la configuración arquitectónica final del edificio y las funciones de cada uno de los espacios arquitectónicos que lo forman. Con ello, se pretende ilustrar la política misional desarrollada por la orden dominica en el proceso de evangelización de la región oaxaqueña.
\end{abstract}

Uno de los capítulos más interesantes de la historia del arte novohispano del Quinientos lo constituyen los complejos conventuales construidos por las órdenes mendicantes como centros para la conversión y evangelización de la población autóctona. Dejando a un lado el foco de la ciudad de México-Tenochtitlán que presenta unas características propias, al imbricarse de una forma más compleja los distintos estamentos de poder, en el resto de poblaciones el recinto conventual fue el núcleo organizativo y funcional que materializaba el dominio de la Monarquia Hispánica sobre el territorio, especialmente en los pueblos de indios de las regiones más alejadas de la capital virreinal.

El presente estudio se centra en el análisis del convento de la orden de predicadores de San Pedro en la ciudad de Etla, estado de Oaxaca. Sus distintas etapas de construcción, la configuración arquitectónica final del edificio, así como las funciones de cada uno de los espacios arquitectónicos que lo configuran, nos ayudarán a ilustrar mejor la política misional desarrollada por la orden dominica en el proceso de evangelización de esta demarcación territorial.

\section{Los Dominicos en la región de Oaxaca}

La orden de predicadores llegó a Nueva España el día 23 de junio de 1526 , formada por ocho miembros procedentes de la península y cuatro de 
la vicaría de las Antillas, dotados con privilegios y proyectos para fundar en Nueva España una nueva provincia. ${ }^{1}$ Se instalaron en la ciudad de México hacia el 25 de julio del mismo año, donde fundaron su primera casa pocos meses después de su llegada. ${ }^{2}$ A partir de 1528 se inicia la expansión de la orden por las naciones mexicana (valle de México y actuales estados de México, Morelos y Puebla), mixteca (zonas de los estados de Puebla y Oaxaca), zapoteca (estado de Oaxaca), así como en Chiapas y Guatemala. ${ }^{3}$

Como señala el investigador Miguel Angel Medina, existió una planificación previa en la ubicación de las casas dominicas. Dicha planificación potenciaba fundamentalmente la región oaxaqueña, por lo que muchas de las casas ubicadas en el valle de México y en la región de Puebla, tenían como finalidad estructurar una cadena de asentamientos que permitieran comunicar directamente la ciudad de México con Oaxaca. ${ }^{4}$ La razón de esta elección se encuentra en que era un territorio extenso que no había sido todavía cristianizado por ninguna orden, debido a su distancia de la capital y al variado mosaico de grupos étnicos que lo formaban, cada uno con su propia lengua. Todo ello nos lo narra fray Francisco de Burgoa en su obra Palestra Historial:

“... y los indios vivían apartados expuestos a las costumbres de idolatrías, y errores en que sus progenitores los habían criado, sin que el temor siquiera pudiese reprimirlos, así porque no había quien se dedicarse a aprender sus idiomas, como porque el retiro, y soledad de las montañas en que vivían hacían intratable su comunicación, y era muy digno de llorar que se perdiesen tantas almas en manos del lobo, habiendo perros que lo arredrasen, y mirasen por aquel copioso rebaño, y lo trajesen al aprisco de la iglesia". ${ }^{5}$

En el año 1529 los frailes se encuentran ya asentados en la ciudad de Oaxaca y, desde esta fecha hasta 1538 aproximadamente, ${ }^{6}$ su labor en la zona se limitó a realizar un primer contacto con los naturales y a localizar los lugares más adecuados para instaurar doctrinas.

1 Ulloa, Daniel: Los predicadores divididos. Los dominicos en Nueva España, siglo XVI, México, 1977, pág.93.

2 Sólo el vicario fray Tomás de Ortiz llegó a la ciudad con antelación, el día 2 de julio, acompañando al licenciado Ponce de León. Fernández Rodríguez, Pedro: Los dominicos en la primera evangelización de México, Salamanca, 1994, págs. 95 y 96.

3 Ibídem, pág. 132.

4 Medina, Miguel Angel: Los dominicos en América: presencia y actuación de los dominicos en la América colonial española de los siglos XV-XIX, Madrid, 1992, pág.72.

5 Burgoa, Francisco: Palestra Historial, México, 1934, pág.28.

6 Pita Moreda, María Teresa: Los predicadores novohispanos del siglo XVI, Salamanca, 1992, pág. 116. 
Entre 1538 y 1578 se produjo la gran expansión dominica por Oaxaca, lo que requería un esquema de organización interna que se basó en dos tipos de asentamientos: prioratos o conventos urbanos y vicarías o conventos rurales, estos últimos localizados en comunidades indígenas. ${ }^{7}$ Esta organización se desarrolló en dos focos culturales y poblacionales diferenciados, por un lado la región mixteca y, por otro, la zapoteca, siguiendo el esquema territorial prehispánico.

En la mixteca se estructuró una cadena de asentamientos que permitieron comunicar la ciudad de México con Oaxaca. El centro principal fue el monasterio de Teposcolula, desde el que se comenzaron las fundaciones hacia el sur en los valles de Achiutla y Tlaxiaco; hacia el norte con la casa de Coixtlahuaca; y hacia el noroeste con los establecimientos de Tonalá y Tamazulapan. ${ }^{8}$ En la zapoteca, aunque la expansión fue menos uniforme, ya que algunos establecimientos estaban dispersos, se siguieron unas líneas directrices hacia el este con centro en la villa alta de San Ildefonso; sudeste para conectarse con la provincia de San Vicente de Chiapas y donde la casa más antigua era la de Tehuantepec; y sur con centros como Huaxolotitlán y Coatlán. ${ }^{9}$ El punto de unión entre ambos núcleos fue la casa matriz de la ciudad de Oaxaca. De esta forma, para 1585 la orden tenía sistematizada la evangelización de toda la región, contando con 17 casas en la mixteca y 18 en la zapoteca. ${ }^{10}$ Esta importancia determinó que, en 1592, la custodia dominica de Oaxaca alcanzara el rango de provincia independiente, convirtiéndose en la Provincia de San Hipólito de Oaxaca.

\section{San Pedro Etla: Historia del edificio}

El convento de San Pedro Etla, ubicado en la región zapoteca, es un claro ejemplo de arquitectura de la evangelización levantada por los dominicos en las vicarías. Estas eran conventos rurales que tendían a ser autosuficientes y contaban con pocos religiosos, entre dos y seis; los cuales asistían a los naturales de su localidad y de los pueblos comarcanos, estos últimos denominados como visitas. Su actividad se centraba en la evangelización, en la cura de almas y en los trabajos de aculturación, tratando de

7 Pita Moreda, María Teresa: "La expansión de la orden por Nueva España", Los dominicos y el Nuevo Mundo. Actas del primer congreso internacional, Sevilla, 1988, pág. 209.

8 Pita Moreda: Los predicadores..., págs. 116 y 117.

9 Ibídem, pág. 118.

10 Ibídem, pág. 119. 
introducir en las poblaciones indígenas los esquemas, planteamientos y formas de vida de los españoles. ${ }^{11}$

La primera construcción de esta zona data de la década de $1530^{12} \mathrm{y}$, según narra fray Francisco de Burgoa en su crónica Geográfica descripción, se levantó en la población cercana de la Natividad:

“... la primera que edificaron fue abajo como quinientos pasos de donde está hoy, y en lo más fértil del pueblo, que se llama Natividad, donde llegan los riegos, y humedades de lo que se vierte; la casa era pequeña y mal labrada porque fue de las primeras donde entraron los religiosos". ${ }^{13}$

Las primeras construcciones destinadas a la evangelización de la población autóctona estaban constituidas por iglesia, una pequeña casa cural y un atrio. Se realizaban con materiales perecederos y tenían un carácter de provisionalidad, debido a la urgencia que requería iniciar las actividades de conversión, a la falta de conocimiento del entorno geográfico y cultural, y a la carencia de artífices tanto indígenas como españoles capaces de proyectar edificios conventuales permanentes. Esta situación es narrada por Burgoa:

"en la forma que el tiempo, y la necesidad permitía en la poca disposición que tenían los indios para labrar los materiales, y en la mucha estrechez de los religiosos contentándose con tan humildes, y pobres casas de tierra, adobes, o tapias mal formadas, y peor cubiertas como hasta hoy parecen en algunos tercios que han quedado". ${ }^{14}$

En la reunión capitular de la orden del año 1550 fue aceptado el convento de Etla como casa fija dominica. ${ }^{15}$ En este momento debió plantearse la construcción de un recinto de carácter permanente acorde con el nuevo rango que adquiría el asentamiento. Sin embargo, no es hasta el año 1570 cuando aparecen las primeras noticias sobre la edificación de un nuevo convento en la localidad, al ser librados por el virrey don Martín Enriquez 600 pesos de oro común a repartir en dos años para la fábrica del nuevo edificio. ${ }^{16}$

11 Ibídem, pág. 205.

12 Gerhard, Peter: Geografía histórica de la Nueva España, 1519-1821, México, 1986, pág.91.

13 Burgoa, Francisco: Geográfica descripción, México, 1934, tomo 2, pág.3.

14 Ibídem, págs. 88 y 89.

15 Mullen, Robert: Dominican Architecture in Sixteenth Century Oaxaca, Arizona, 1975, pág.38.

16 AGN, Hospital de Jesús, 208, Traslado del mandamiento dado por el virrey, don Martín Enríquez, para que se paguen 600 pesos de oro común a los naturales de la ciudad de Etla, para la construcción de su monasterio. México, 20 de febrero de 1570, documento 1. 
Según dicho documento, la construcción se plantea porque el primer edificio de adobes y paja había sufrido graves daños tras un reciente temblor. Como primer paso para su realización fue enviada al virrey una traza que resultó no ser moderada, es decir, se había presentado un proyecto de grandes dimensiones y suntuosidad, en relación con los medios económicos y humanos disponibles por la población. Por esta razón, tras haber sido examinada, la traza fue modificada por Claudio de Arciniega, maestro mayor de las obras de cantería de la Nueva España, estableciendo una serie de variaciones encaminadas a racionalizar los recursos disponibles.

Mientras se iniciaba este nuevo convento, se mantenía en uso el anterior, cuya provisionalidad fue motivo de un desagradable accidente. El hecho ocurrió siendo vicario fray Alonso de la Anunciación, por lo que se sitúa en el año 1575, y fue el derrumbe de una de las construcciones durante la celebración de la procesión del Corpus, que acabó con la vida de este fraile. Fray Francisco de Burgoa nos cuenta lo sucedido:

"Iba llegando la solemnidad en que lucir el aparato de la fiesta del corpus, en que sale debajo de aquellas especies en procesión a santificar las calles, y para la entrada al patio de la iglesia, era la representación, no había capilla, ni lugar decente en su desabrigo, y se trató de hacer un largo corredor cubierto, y de malos fundamentos, y peores maderas, como de prestado, y apriesa acabosé sin tiempo para secarse.... el buen vicario gustó de salir revestido con la custodia en las manos el día de la fiesta; el concurso de la gente a la novedad era inmenso, y entrando con sus compañeros revestidos, debajo del corredor que estaba decentemente colgado, y prevenido altar donde poner la custodia, y adorado el Divinisimo Sacramento,.... fue tanta la gente que se subió sobre la cubierta del corredor por gozar de la comedia, que sin dar lugar a quitar la custodia, se postro el pretil dando de pechos y cara abajo,.. fue cayendo la cubierta toda de vigas y terrapleno". ${ }^{17}$

Este desafortunado suceso fue el que, según Burgoa, originó el abandono de la primitiva iglesia y la construcción del nuevo recinto "en lo más firme y macizo sobre canteras espaciosas de piedra". ${ }^{18}$

El 12 de marzo de 1580 los naturales de Etla vuelven a solicitar al virrey que les sea concedida ayuda para alzar y reparar su iglesia, pues ésta se les había caído. ${ }^{19}$ Pocos años después, concretamente hacia 1595 , la armadura de madera de la cubierta del templo estaba todavía en construc-

17 Burgoa: Geográfica..., tomo 2, pág.4.

18 Ibídem, tomo 2, pág. 5.

19 AGN, General de Parte, 2, 632, al alcalde mayor de Antequera para que informe sobre el estado de la iglesia y ornamentos de que tiene necesidad el pueblo de Etla. México, 12 de marzo de 1580 , documento 3 . 
ción, siendo el carpintero español Sebastián García, junto con un grupo de indígenas, los encargados de su ejecución. ${ }^{20}$

En la última fase del convento se levantaron el resto de dependencias monásticas. El claustro se edificó bajo el prior fray José Calderón, es decir, entre 1612 y 1619, mientras que el resto del recinto se concluyó bajo el priorato de fray Alonso de Espinosa hacia 1636. ${ }^{21}$

La configuración original del recinto conventual ha sufrido diversas modificaciones a lo largo de los siglos. Hoy día no es posible contemplar la cubierta original de madera de la iglesia porque está techada de concreto desde el año $1961 .{ }^{22}$ También la barda del atrio está reconstruida y ha perdido tanto la capilla abierta como las capillas posas que poseía.

\section{Configuración arquitectónica: espacios y funciones}

La traza del complejo conventual de San Pedro Etla sigue el esquema constructivo de la mayoría de los conventos mendicantes novohispanos del Quinientos. Esta homogeneidad arquitectónica fue debida a varios factores de gran importancia. El primero de ellos fue el papel que jugó la Corona española, la cual, gracias a la prerrogativas del patronato real, tenía el derecho y la obligación de controlar el desarrollo eclesiástico en el Nuevo Mundo y, por consiguiente, supervisar las obras arquitectónicas religiosas. Además, la Corona financió parcial o totalmente la construcción de los conventos mendicantes, razón por la cual sus derechos de intervención estaban totalmente justificados, como veremos posteriormente.

Esta política intervencionista estuvo en manos de los virreyes, quienes jugarán un papel de primer orden en la designación y erección de los conjuntos mendicantes. En este sentido debemos destacar la figura de don Antonio de Mendoza, quien concertó la llamada "traza moderada" con los religiosos franciscanos y agustinos, aconsejando a su sucesor en el cargo, don Luis de Velasco, que hiciera lo mismo con la orden de Santo Domingo. ${ }^{23}$

20 Kubler, George: Arquitectura mexicana del siglo XVI, México, 1982, pág.155. Burgoa, Francisco: Geográfica..., tomo 2, pág.5.

21 Kubler: Arquitectura..., pág.632.

22 Vences Vidal, Magdalena: "Notas para la arquitectura de la evangelización en el valle de Oaxaca", Los dominicos y el Nuevo Mundo. Actas del segundo congreso internacional, Salamanca, 1990, pág.506.

23 Colección de documentos inéditos relativos al descubrimiento, conquista y colonización de las posesiones españolas en América y Oceanía, sacados del real Archivo de Indias, volumen VI, Madrid, 1864-1885, págs.33 y 34. 
El segundo factor fue la homogeneidad en la actividad evangelizadora que desarrollaron las tres religiones, fruto de la llamada "unión santa" que alcanzaron los mendicantes en 1541, la cual documenta el agustino fray Juan de Grijalva:

"Hicieron las religiones una unión santa, que así la llamó el señor obispo Zumárraga y por tal la juzgó todo el reino, y fue, que para todo lo que hubiesen de hacer, así en lo tocante a la conversión de los naturales, como a la administración de los santos sacramentos y en las costumbres en que hubiesen de imponer a los tales naturales, hubiese uniformidad: de manera que todas caminasen a un paso. Fue importantísima esta unión, para la fundación de estas iglesias". ${ }^{24}$

El tercer y último factor fue la falta de maestros y oficiales cualificados para la construcción de los recintos conventuales. La traza y supervisión sobre el terreno de las obras estuvo a cargo, la mayoría de las veces, de los propios frailes de las órdenes mendicantes. Posteriormente, dicha traza debía ser aceptada y, si era necesario, modificada por el maestro mayor de obras de la ciudad de México, nombrado directamente por el virrey. Este es el caso de Claudio de Arciniega quien, primero como maestro mayor de las obras de cantería de la Nueva España y, posteriormente, como alarife de la ciudad de México, superviso prácticamente todas las trazas y construcciones realizadas en el virreinato entre 1560 y $1593 .{ }^{25}$ Así, inspeccionó las obras de la catedral de Pátzcuaro, trazó y dirigió las obras de la catedral de México, intervino en la construcción de la catedral vieja de Puebla, de la iglesia de San Antón y de los conventos de San Francisco, Santo Domingo y San Agustín de la ciudad de México, en el hospital real de los naturales y de los convalecientes también en dicha ciudad, trazó el fuerte de San Juan de Ulúa, y se ocupó del desagüe de las minas de Taxco. ${ }^{26}$

El convento de Etla está compuesto por tres unidades arquitectónicas básicas: el atrio con sus distintos elementos, el templo y las dependencias monásticas. El atrio es el espacio natural, limitado por una barda de piedra, que antecede al templo y encierra al resto de espacios conventuales. Actualmente, el atrio es de planta rectangular, delimitado por una barda de

24 Grijalva, Juan: Crónica de la orden de nuestro padre San Agustín en la provincia de Nueva España, México, 1985, págs.114 y 115.

25 Castro Morales, Efraín: "Los maestros mayores de la catedral de México", Artes de México, núm.182-183, México, 1974, pág.139.

26 Sobre la significación de Claudio de Arciniega y su obra, véase Manuel Toussaint: Claudio de Arciniega, arquitecto de la Nueva España, México, 1981, y Marco Dorta, Enrique: "Claudio de Arciniega, arquitecto de la catedral de México", Actas del XXIII congreso internacional de historia del arte entre el Mediterráneo y el Atlántico, Granada, 1976, págs. 351-360. 
mampostería, y arcada de acceso en el eje longitudinal de la fachada de la iglesia conventual, la cual comunica dicho atrio con la plaza pública de la localidad. Esta disposición convirtió a ambos espacios abiertos en el centro neurálgico de la población, donde estaban representadas las instituciones y actividades más emblemáticas de la comunidad.

Además, la explanada abierta del atrio articulada con la capilla abierta y las capillas posas se transformaba en el centro ceremonial de la población indígena. La capilla abierta de Etla se ubicaba, según el investigador Robert Mullen, junto a la puerta norte del templo, ${ }^{27}$ es decir, en una localización semejante a la del convento de Coixtlahuaca, lo que originaría, en nuestra opinión, un característico atrio en forma de L. Desgraciadamente, ni la capilla abierta, ni las capillas posas se conservan, pero sabemos de la existencia de estas últimas por la descripción que realiza Burgoa:

“.. el patio de la iglesia está muy bien cercado y almenado, tiene cuatro capillas muy capaces de tixera en los ángulos para las procesiones". ${ }^{28}$

En Etla, como en todos los conventos mendicantes, la articulación atrio, capilla abierta y capillas posas fue el recinto donde se desarrollaron todas las actividades relacionadas con la cristianización de la población autóctona. Por ejemplo, la capilla abierta funcionaba como iglesia de indios y escuela (instrucción doctrinal, primeras letras, formación profesional); las capillas posas como altares para las procesiones y recintos para la educación catequística; y la conjunción de los tres elementos como espacio ceremonial donde se celebraban danzas, representaciones teatrales, procesiones, etc. Por tanto, esta unidad constructiva de los complejos conventuales, fue un espacio esencial para la conversión y evangelización de los naturales, pues unía el concepto cultural mesoamericano de culto extrovertido con los contenidos teológicos cristianos occidentales. ${ }^{29}$

El templo, como recinto sagrado de toda la comunidad religiosa, era el centro del convento. En Etla es de una sola nave con cuatro tramos, presbiterio diferenciado de planta cuadrada y coro a los pies. ${ }^{30}$ Pero, además, presenta contrafuertes interiores que originan tres capillas en cada uno de

27 Mullen: Dominican Architecture..., pág.6.

28 Burgoa: Geográfica..., tomo 2, pág.7.

29 Para una mayor profundidad del tema, véase Espinosa Spínola, Gloria: Arquitectura de la conversión y evangelización en la Nueva España durante el siglo XVI, Almería, 1999.

30 Mullen: Dominican Architecture..., pág. 84. Este investigador señala que esta forma del presbiterio es una de las características más sobresalientes de las iglesias dominicas de la zapoteca. 
los muros laterales de la iglesia. La existencia de estas naves da lugar a un tipo de iglesia que Kubler llamó "criptocolateral" y que se caracteriza porque "en dichos templos, los pasillos laterales están ocupados por una hilera de capillas que lo hacen desaparecer como volumen efectivo, siendo distinguibles únicamente desde el exterior. De esta manera, los templos criptocolaterales son de una y de tres naves a la vez". ${ }^{31}$

En Etla esta concepción espacial criptocolateral puede observarse tanto en la planta como en el alzado exterior del edificio: sillares de piedra forman una volumetría simple y geométrica con ventanales abiertos en la parte superior, destacándose el gran cubo que forma la nave que es completado, en ambos laterales, por un contrafuerte corrido de poca profundidad que aloja interiormente las capillas laterales.

La iglesia se completaba con una cubierta de madera, que según Burgoa era:

"Toda de artesones sobre tisera de tanto arte, y curiosidad, que sin verse las vigas es todo el techo de ochavados de una tercia de hueco, guarnecidos de molduras que hacen ingeniosa labor como si fuera de cera". ${ }^{32}$

El resto del templo se cubre con bóvedas de medio cañón para las capillas laterales y bóveda baída para el coro.

En nuestra opinión, la planta criptocolateral comparte la concepción espacial de la traza moderada, que no había sido respetada por el primer proyecto enviado al virrey en 1570 . Este documento describe una iglesia con crucero y totalmente cubierta de bóveda, por lo que requería unas paredes excesivamente gruesas. La revisión de Claudio de Arcinega altera la traza, sustituyendo la cubierta de piedra por otra de madera y eliminando el mencionado crucero. Con esta forma se adaptaría al templo mendicante que se desarrolla a partir de 1550 , el cual debía responder a las premisas de la traza mendocina: templo de una sola nave orientado de este a oeste, con presbiterio diferenciado de forma rectangular o poligonal, coro a los pies y doble portada.

Esta configuración arquitectónica del templo evitaba la suntuosidad en las obras y permitía un mayor control de los gastos que realizaba la Corona en la construcción de los recintos conventuales. ${ }^{33}$ Así, por ejemplo,

31 Kubler: Arquitectura..., pág.243.

32 Burgoa: Geográfica..., tomo 2, pág.5.

33 García-Abasolo, Antonio F.: Martín Enríquez y la reforma de 1568 en la Nueva España, Sevilla, 1983, pág. 304. 
a fin de evitar el empleo de la contribución oficial en construcciones menos necesarias para el funcionamiento de los templos, la audiencia de México en 1573 suspendió las ayudas que recibían los mendicantes, en tanto se establecía una fórmula para la financiación. La solución definitiva, dada al año siguiente, consistió en que la Corona seguiría contribuyendo, pero aplicando sus asignaciones a la construcción del cuerpo principal y la capilla mayor de las iglesias, mientras que para la financiación de las capillas laterales, los religiosos buscarían la ayuda de personas particulares. ${ }^{34}$ Esta resolución explica por qué en las iglesias conventuales de los pueblos de indios predominan los templos de una sola nave o criptocolaterales de capillas poco profundas como en Etla, los cuales no requieren un gasto adicional para su ejecución.

Además, tanto si se tratan de iglesias de una sola nave como de planta criptocolateral, lo fundamental es que el templo se concibe como una "caja cerrada", en el que el centro referencial y eje de la composición lo constituye el presbiterio, jerarquizado por su situación elevada por varios escalones del pavimento de la nave y la presencia de un arco triunfal. Esta disposición de los templos mendicantes novohispanos mantiene los rasgos de los templos conventuales castellanos, donde ya en la primera mitad del siglo XV se adoptó el modelo llamado de iglesia de predicación, establecida por dominicos y franciscanos durante los siglos XIII y XIV en el sur de Francia, Italia y la Corona de Aragón. Este templo, de carácter eminentemente funcional, es de una sola nave libre de cualquier estorbo visual, debido a la inexistencia de soportes exentos y por quedar las capillas laterales empotradas entre sus contrafuertes, insertas prácticamente en los muros..$^{35}$

Esta concepción arquitectónica, que pretende subordinar todo al recinto del presbiterio, como lugar ocupado por la divinidad, responde a la capacidad de los frailes mendicantes por sintetizar y resaltar los aspectos más destacados del ritual cristiano, a fin de favorecer la mejor compresión y aprendizaje de una comunidad religiosa recientemente convertida. Había que desdeñar lo superfluo y magnificar lo esencial, pues sólo de esta forma serían asimiladas por una población pagana las premisas cristianas.

Etla no es la única iglesia dominica que responde a este tipo de templo, ya que esta planta también la presentan la iglesia de Santo Domingo

34 Ibídem, pág. 304.

35 Marias, Fernando: El largo siglo XVI, Madrid, 1989, pág.119. 
en la ciudad de México, la iglesia de Santo Domingo en Oaxaca, San Juan Bautista Coixtlahuaca, San Pedro Huitzo e incluso la de Santo Domingo Oaxtepec que, aunque es de una sola nave, tiene arcadas poco profundas en los muros laterales, por lo que se acerca a la planta criptocolateral. ${ }^{36}$

La última unidad constructiva es el claustro y sus dependencias anexas, destinadas al uso exclusivo de la comunidad religiosa dominica. El claustro, como señaló Santiago Sebastián, es un centro sagrado o microcosmos, que está construido como una ciudad sagrada: "es la Jerusalén celeste, ese nuevo mundo descrito en el Apocalipsis, en cuyo centro se cruzan las coordenadas espaciales y se señala por un pozo, árbol, fuente o columna, indicando que allí hay un omphalos o centro del cosmos". ${ }^{37}$

En Etla, encontramos un claustro doblado, formado por galerías de arcos de medio punto, que en el primer piso apoyan sobre columnas y en el segundo sobre pilares; ambos tipos de soportes estan adosados a pilares de sección octogonal. Sus cubiertas son bóvedas baídas. ${ }^{38}$ La fuente central y el programa decorativo que cubría las paredes del claustro, consistente en un apostolado, símbolo del cuerpo de la iglesia y de los primeros predicadores de la religión, terminaban de completar la concepción simbólica que hemos señalado anteriormente. De la ornamentación mural sólo se conserva un fragmento de esquina donde, inserto en un recuadro, aparece la representación de San Andrés y Santiago el Mayor en el muro frontal a él. El resto de la composición está realizada a base de elementos vegetales, la cruz florenzada propia de la orden, roleos y candelieri.

En conclusión, el convento de San Pedro Etla, aunque muy modificado por intervenciones posteriores, conserva la traza moderada característica de los establecimientos mendicantes que durante el siglo XVI se erigieron en los centros ceremoniales de los pueblos de indios. Estos recintos representan, en su concepción espacial y simbólica, la unión de los tres estamentos que, relacionándose, formarán el nuevo cristianismo novohispano: la iglesia lo divino, el claustro el estamento mendicante y el atrio con sus dependencias el mundo indígena.

36 Mullen: Dominican Architecture..., págs. 67-91

37 Sebastián López, Santiago, Mesa Figueroa, Juan, Gisbert de Mesa, Teresa: Arte Iberoamericano, desde la colonización a la independencia, Suma Artis, volumen 28, Madrid, 1985, pág. 134.

38 Kubler: Arquitectura..., pág. 414. Este autor señala que la misma cuadrilla de trabajadores construyó los claustros de Cuilapan y Etla, por lo menos en lo que respecta al primer cuerpo de Etla. 


\section{Documentación}

La reconstrucción histórica del convento de San Pedro Etla se ha basado tradicionalmente en los datos que fray Francisco de Burgoa recoge en sus obras Palestra historial y Geográfica descripción que vieron la luz en el año 1674. Aunque la información que ofrecen es de sumo interés, ésta se completa con los tres documentos que transcribimos a continuación y que aclaran la evolución constructiva del edificio durante el siglo XVI.

Documento 1. AGN, Hospital de Jesús, 208. Traslado del mandamiento dado por el virrey don Martín Enríquez para que se paguen 600 pesos de oro común a los naturales de la ciudad de Etla (Oaxaca) para la construcción de su monasterio. México, 20 de febrero de 1570.

Los juezes e ofiçiales de la real haçienda de Su Magestad desta Nueua España, administradores y depositarios de las haçiendas y estados de don Martín Cortes, marqués del ualle, hazemos sauer a Juan Gómez Çorita, vecino de la çiudad de Antequera, a cuyo cargo, por nuestra comisión, está la cobranza de los tributos que los naturales de las dichas villas del dicho estado en el ualle de Guaxaca, son obligados a dar conforme a sus tasaçiones, que por vn mandamiento del muy excelente señor uisorey don Martín Enrriquez, cuyo tenor es este que se sigue:

Don Martín Enrriquez, uisorrey y gouernador e capitán general por Su Magestad en esta Nueua España y presidente de la audiencia real que en ella reside, por quanto por parte de los religiosos de la horden de Santo Domingo que residen en la çiudad de Etla del estado del marqués del ualle, e por los naturales della, me fue hecha relación que de muchos años a esta parte auia monesterio de la dicha orden en la dicha çiudad, y la yglesia y monesterio auia sido de prestado, hecho de adoves y cubierto de paja, e por ser de ruin edifiçio, con los temblores pasados se auia todo abierto y aruinado de manera que no se podía deçir misa en la dicha yglesia, ni viuir en el dicho monesterio. E que agora ellos querían hazer la dicha yglesia e monesterio de nueuo, y me pidieron mandase que de los tributos que heran obligados a dar al marqués del ualle, cuya hera la dicha villa, mandase ayudarlos para la dicha obra conforme a lo que Su Magestad tiene mandado.

E por mi se cometio a Francisco Montealegre, alcalde mayor de la çiudad de Antequera, para que viese la dicha yglesia y monesterio que al presente está hecho, y entendiese si hera sufiçiente para se poder pasar con ello, o si hera neçesario hazer otro de nueuo, y de que obra y tamaño conbenia hazerse, y en que tiempo, y con quantos yndios se acabaría, y que podría cos- 
tar, y si auia lejos y descuidado los materiales o cerca, e si los dichos yndios de su voluntad lo quieren hazer, y embiase relación de todo, con la traça de la obra que se pensaua hazer; el qual hizo sobrello çiertas diligençias, las quales con su pareçer y las dichas traça firmado de su nombre lo ynbio ante mi, y para más justificaçión y entender si la obra que se pretendía hazer conforme a la dicha traça hera moderada, Claudio de Arçiniega, maestro de semejantes obras, el qual ansi mismo dió pareçer que la dicha obra conforme a la dicha traça hera moderada con que la cobertura della fuese de madera y no de piedra, que la yglesia no lleuase cruzero, como en la dicha traza benía trazado y por señalado, y que las paredes se adelgazen y no sean tan gruesas, y que las dos pieças que la vna sirue de despensa y la otra de secrestas se desbien ocho pies a vn lado del quarto haçia la parte del mediodía, para que se pueda hazer vna bentana para dar luz al ambulatorio que está en el dormitorio, y se aga otra ventana en la otra parte frontera desta para lo mesmo, e fecho de manera que la una esté a lebante y la otra a poniente.

Y por mi visto el dicho pareçer, por la presente, doy licencia y facultad para que la dicha yglesia y monesterio se aga conforme a la dicha traça que va firmada del dicho Francisco Montealegre y del secretario yuso escripto, guardando la horden y paresçer que dió el dicho Claudio de Arçiniega de que arriba se haze relación. Y para ayuda de lo que se a de gastar en la dicha obra por tiempo de dos años, se dé a los dichos yndios seisçientos pesos de oro común, tresçientos en cada vno de los dichos dos años, atento a lo qual por la presente mando a los juezes ofiçiales de la real haçienda de Su Magestad a cuyo cargo es el beneficio e cobranza de los tributos e bienes del estado del dicho marqués, que dé qualesquier maravedis e pesos de oro que son o fueren a su cargo de los tributos que son obligados a dar los yndios de la dicha villa, los den o tomen en quenta los dichos seisçientos pesos, trezientos en cada vno de los dichos dos años; obligándose el gouernador, alcaldes, regidores y mayordomo de la dicha villa que los gastarán en la dicha obra, y darán quenta en que y como los gastaron cada y quando se les pida, y dandóselos y pagandóselos con este mandamiento y su carta de pago, y la dicha obligación, tomada la razón y la contaduría de dicho estado mando se les resçiba y pase en quenta, hecho en México a diez días del mes de enero de mill y quinientos y setenta años, don Martín Enrriquez. Por mandado de su excelencia Juan de Cueua.

Que originalmente queda en la dicha contaduría, se nos manda que dé los maravedies e pesos de oro que son a nuestro cargo de los tributos que los naturales de la dicha villa de Etla son obligados a dar, demos y paguemos al gouernador, alcaldes, regidores y prinçipales della seisçientos pesos de oro común en dos años, trezientos en cada vno, para la obra y edifiçio del monesterio e yglesia de la dicha villa, por ende de los maravedis y pesos de oro que 
son o fueren a vuestro cargo de los que por los dichos naturales son obligados a dar en tributo les dad y pagad, o les tomareis en quenta dellos, los dichos seisçientos pesos de oro común en los dichos dos años, treçientos pesos en cada vn año, que corran desde el día que les hiçieredes la primera paga, obligándose ante todas cosas el dicho gouernador, alcaldes y regidores y mayordomo de la dicha villa que gastaran los dichos seisçientos pesos de oro en la dicha obra, e daran quenta en que y como los gastaron, cada y quando se les pida que con su carta de pago y la dicha obligación y este reciuimiento o su treslado signado de escriuano les serán rresçiuidos en quenta. Fecho en México a veynte de febrero de mill y quinientos y setenta años.

Alonso de Villanueva (rúbrica), Juan de Cueua (rúbrica), Gregorio Díaz Casas, escriuano público (rúbrica), Gerónimo de Albornoz (rúbrica).

Para que Juan Gómez Çorita dé a los naturales de la ciudad de Etla seisçientos pesos de oro común en dos años, treçientos en cada vno, para la obra del monesterio y yglesia de la dicha villa, obligándose a dar quenta dellos.

Documento 2. AGN, Hospital de Jesús, 208. Pago de Juan Gómez Zorita a los naturales de Etla (Oaxaca) de 300 pesos para la obra de su monasterio. Antequera, 1 de diciembre de 1573.

En la ciudad de Antequera desta Nueva España, primero día del mes de diziembre de mill e quinientos y setenta y tres años, don Miguel de León, gouernador, e Matheo Ruiz e Domingo de Sosa, alcaldes, Juan Ferrez, Julián Carrasço e Francisco Vázquez, yndios regidores de la villa de Etla del estado del marqués del valle, a los quales yo el escriuano yuso escripto doy fe que conozco, mediante y por lengua de Pedro Maçias, ynterprete de lengua mexicana que juro a Dios e a vna señal de la cruz e prometio de ynterpretar verdad, dixeron que an rreçibido de Juan Gómez Çurita, vezino desta dicha çibdad a cuyo cargo está la cobrança de los tributos e hazienda del dicho marqués, por comisión de los señores juezes oficiales que por Su Magestad rresiden en la çibdad de México, trezientos pesos de oro común que son de la segunda paga, a cumplimiento de seisçientos pesos de dicho oro que su excelencia el señor virrey le mandó dar de los tributos que los naturales de la dicha villa pagarán al dicho marqués, para la obra de la yglesia e monasterio della, como paresçe por el mandamiento executoria y recaudo desta otra parte, porque los otros trezientos pesos de la primera paga se los tenía dados, de que le aviamos dado carta de pago por manera que con estos trezientos pesos de la postrera paga le ace dicho Juan Gómez acabado de pagar, y de todos seyscientos pesos se hazen cargo para los gastar y emplear en la dicha obra, y dellos darán quenta con pago a quien e como e cuando se le pidan, como 
su excelencia por el dicho mandamiento lo manda, por lo qual obligaron sus personas y bienes y bienes propios e rentas de la comunidad de la dicha villa, e los que supieron firmar lo firmaron, testigos Alonso Trebiño y Juan Gallego y el dicho Pedro Maçias, el qual ansi mesmo lo firmó, y por los que no supieron firmar de los dichos otorgantes firmo a su ruego el dicho Juan Gallego.

Don Miguel de León (rúbrica), Matheo Rodriguez (rúbrica), Juan Fernández (rúbrica), Francisco Várquez (rúbrica), Julián Carrasço (rúbrica), Pedro Maçias (rúbrica), Juan Gallego (rúbrica).

Ante mi Pedro Gutierrez de Urdiçia escriuano publico (rúbrica).

(Margen superior derecha): Etla, sobre la obra y destos 600 pesos están cobrados hasta oy fin de agosto de 1572 los 300, anse de pedir a Juan Gómez los otros 300 .

Documento 3. AGN, General de Parte, 2, 632. Al alcarde mayor de Antequera para que informe sobre el estado de la iglesia y ornamentos de que tiene necesidad en el pueblo de Etla. México, 12 de marzo de 1580.

$<$ Los de Etla $>$ (margen izquierdo).

Don Martín Enrriquez, virrey, hago sauer a vos el alcalde mayor de la ciudad de Antequera que, por parte de los naturales del pueblo de Etla del estado del marques del valle, me ha sido fecha relacion que la yglesia del dicho pueblo se nos ha caydo y no tienen donde se selebren los diuinos oficios e oygan misa, por nesesidad que tienen no la han alçado y reparado, y me pidieron mandase dar horden como los açedores del dicho marques les socorra con alguna cosa para ayuda del dicho reparo y con algunos ornamentos y otras cosas de que tiene la dicha yglesia nesesidad, que de muchos años a esta parte por parte del dicho marques no se le ha dado socorro ni cosa alguna de lo suso referido. Y por mi vista por la pressente os mando que luego queste mi mandamiento vos sea mostrado os ynformeis e auerigueis el estado que esta la dicha yglesia y los ornamentos y otras cosas que para ello tienen nesesidad e que cantidad de pesos de oro sera menester para uno y lo otro, lo qual bereis personalmente y dello me ynformareis y enbiareis relacion juntamente con vuestro parecer jurado en forma. Fecho en Mexico a doze dias del mes de março de myll e quinientos y ochenta años. Don Martin Enrriquez por mandado de su excelencia Martin Lopez de Gauna, refrendado por Juan de Cueba. 